Palimpsesto Vol. 11, No 18 (enero-junio, 2021): 91-100

Universidad de Santiago de Chile, ISSN 0718-5898

\author{
Valeria Sánchez Prieto \\ Semillero de Investigación de Estudios Estructuralistas. Pontificia Universidad Javeriana \\ sanchez.valeria2922@gmail.com
}

Juan Camilo Ospina Deaza

Semillero de Investigación de Estudios Estructuralistas. Pontificia Universidad Javeriana

jospinad@javeriana.edu.co

\title{
Museo y Antropología. El saber antropológico en la construcción de nación en Colombia*
}

\section{Museum and Anthropology. The Anthropological knowledge in the Nation Building in Colombia}

\begin{abstract}
Resumen
Así como la antropología ha proveído conocimientos sobre la diversidad cultural del país no sólo por las líneas de la academia, el museo se encuentra como canal de comunicación con el público que visita las colecciones. En consecuencia, en el campo museal la antropología, junto con otras disciplinas, ha sido comprendida como un saber-poder en el proceso de construcción de nación. Este análisis busca contribuir al entendimiento del papel de la antropología en la formación de nación a través de los museos. Por este motivo, en este artículo reflexionamos sobre cómo la narrativa de nación, que atraviesa las colecciones de algunos museos del país, está determinada por la apropiación de elementos del saber antropológico por parte del discurso oficial de Estado. Lo anterior bajo la premisa de que, el museo se caracteriza por realizar 'actos de estado' por medio del ejercicio de clasificación y regulación de sujetos, objetos, lugares, prácticas, experiencias y acontecimientos.
\end{abstract}

Palabras claves: museo, antropología, construcción de nación, saber de estado.

\footnotetext{
* Esta reflexión es uno de los productos del análisis al campo museal en Colombia, presentado en la tesis de pregrado en antropología titulada "El museo como dispositivo de poder. Construcciones simbólicas en torno al proceso de paz en Colombia" (2019). Además, el primer borrador de este artículo fue presentado como ponencia en el XVII Congreso de Antropología en Colombia (2019), en el simposio: Las condiciones de posibilidad de la producción, circulación y consumo del conocimiento antropológico en la sociedad contemporánea. El cual fue realizado en la Universidad ICESI de Cali-Colombia. 


\begin{abstract}
Just as Anthropology has provided knowledge about the Colombian cultural diversity not only along the lines of the academy, the museum is a communication channel between the public and the museum collections. In consequence, in the museal field anthropology, with other disciplines, has been comprehend as a power-knowledge in the process of nation building. This analysis seeks to contribute to the understanding of the anthropology role in the nation formation through museums. For this reason, in this article we reflect about how the nation's narrative is determined in some museums by the appropriation of anthropological knowledge by the state official discourse. The above, under the premise that the museum works as a state agent in charge of doing 'state acts' through the exercise of classification and regulation of populations.
\end{abstract}

Keywords: Museum, Anthropology, Nation building, State knowledge.

La antropología como saber tiene mucho que decir sobre la diversidad cultural, el pasado prehispánico, la simbolización de la vida social, los diálogos interculturales, etc., y para difundir este saber no sólo por las líneas de la academia, se encuentra el museo como canal de comunicación con el público que visita las colecciones. En muchos espacios, el museo se ha caracterizado por ser un dispositivo pedagógico-educativo que busca enseñar al público, los valores que se destacan en la construcción de nación. En este artículo se reflexiona cómo la narrativa de nación, que atraviesa las colecciones de algunos museos del país, está determinada por la apropiación de elementos del saber antropológico por parte del discurso oficial de estado. El análisis presentado es producto de una investigación sobre el campo museal en Colombia.

Autores como Myriam Jimeno (1993), Marcela Echeverry (1997), Roberto Pineda Giraldo (1999) (2005), Roberto Pineda Camacho (2008) y otros, han realizado importantes análisis sobre la historización del campo de la antropología en Colombia. Sus aportes van desde las fechas de surgimiento de la disciplina, la influencia de reconocidos científicos sociales, organizaciones estatales y privadas en torno a su emergencia, las transformaciones de los ejes temáticos que aborda este saber, entre otros componentes. Ospina menciona como también

existen trabajos que han aplicado otras técnicas de investigación sobre el campo, tales como: Zambrano y Durán (2017), Restrepo (2006) entre otros. Todos estos estudios han servido para complejizar el que hacer antropológico, ya sea al conocer las tendencias temáticas en la historia de la antropología, conociendo las relaciones de centroperiferias en nuestro quehacer científico o profundizando en las trayectorias vitales de los egresados de antropología. (Ospina, 2018, p.13)

No obstante, en el estado del arte sobre la profesionalización de la antropología en Colombia, encontramos que no existe una amplia bibliografía sobre investigaciones acerca de las dinámicas particulares que caracterizan el ejercicio de la antropología en el mercado laboral. En la actualidad se está abriendo un espacio de indagación por los usos sociales de la antropología y de los antropólogos. Los trabajos de Ospina (2018), Letona (2016), Restrepo (2017), Zambrano y 
Duran (2017), y Buenaventura (2008) concuerdan en que "creer que los temas de antropología solo se producen por la antropología misma es desconocer el contexto laboral y económico de que la antropología es un saber-poder, utilizado por distintos sectores sociales como el Estado, el mercado y otros, para sus propios fines" (Ospina, p.62).

Como una agenda investigativa a futuro, sería indispensable conocer la posición que ocupan los profesionales en antropología en el campo laboral en Colombia. Analizar las trayectorias laborales de los egresados permitiría conocer las lógicas de empleo y los niveles de profesionalización. Esto porque, así como Escobar afirma, "es claro el hecho que los antropólogos trabajan bajo un contexto político y epistemológico que configura tanto sus prácticas como sus objetos de estudio que esta fuera de su control" (Escobar 1993, p.378). En este sentido, viendo la necesidad de conocer cuáles son los usos sociales de este saber en la sociedad, este análisis busca contribuir al entendimiento del papel de la antropología en la formación de nación en los museos del país.

Siguiendo a Emile Durkheim, "la vida social debe explicarse, no por la concepción que de ella tienen los que en ella participan, sino por causas profundas que escapan a la conciencia" (1982, p.30). A razón de lo anterior, se deben analizar las dinámicas de mercado, las dinámicas de poder y los procesos de subjetivación que influencian a la antropología. En consecuencia, este artículo busca ser un aporte al conocimiento sobre el quehacer antropológico en el campo museal en Colombia.

A continuación, resaltaremos en primer lugar algunos aspectos de la profesionalización de la antropología en Colombia en relación con la emergencia de los museos en el país; en segundo lugar, observaremos la influencia del saber antropológico en la creación de las narrativas que permean las colecciones museales; y, finalmente reflexionaremos sobre el rol de la antropología como un saber de estado ${ }^{1} \mathrm{y}$, el papel de los museos en la formación de nación.

Durante la década de 1940 tuvo lugar la profesionalización de la antropología en Colombia en el contexto de la República Liberal, periodo en el cual se promovía un proyecto de modernización nacional cuyo énfasis era la consolidación del Estado. Siguiendo a Marcela Echeverry "es importante considerar que lo que he denominado un proceso de profesionalización, implica precisamente la afiliación entre un tipo de conocimiento y los intereses -del Estado; la profesionalización de un conocimiento es la legitimación por vías institucionales de su práctica, dándole un lugar privilegiado en el mercado de las ocupaciones" (Echeverri, 1997, p.67).

Las ciencias sociales, entre ellas la antropología, fueron creadas "con el fin de suplir unas demandas que el proyecto de modernización estatal estaba requiriendo" (Ospina, p.134). Según Roberto Pineda la antropología durante esta década tenía "un propósito que podría muy bien denominarse etnografía de salvamento: registrar el contenido cultural de las comunidades indias, que corrían el riesgo de desaparecer por extinción de sus miembros o por procesos de incorporación a la vida nacional" (Pineda, 2005, p.1).

La información recolectada por antropólogos y arqueólogos sobre la diversidad cultural del país, va a ser luego retomada, entre otras instituciones, por los museos para crear una

\footnotetext{
${ }^{1}$ Siguiendo los postulados de Pierre Bourdieu, "lo que nosotros llamamos estado, lo que señalamos confusamente cuando pensamos en el estado es un tipo de principio de orden público, entendido no sólo en sus formas físicas evidentes sino también en sus formas simbólicas inconscientes, las cuales aparentemente son profundamente autoevidentes. Una de las funciones más generales del estado es la producción y canonización de las clasificaciones sociales" (2014, p.9). Por lo tanto, el estado no es un sujeto con un tipo de agenciamiento particular, el estado "no es un bloque, es un campo" (p.20). En este sentido, no nos referiremos al estado en letras mayúsculas.
} 
narrativa colectiva sobre sujetos, objetos y prácticas particulares. De allí que Amada Pérez afirme que, los museos se convirtieron en recintos a través de los cuales se promovía la difusión de la ciencia y las artes con el fin de "moldear a los ciudadanos creando una imagen de nación a través de la historia y la etnografia" (2010, p.86).

Conocido por agrupar y coleccionar un conjunto de objetos con características particulares, el museo se diversificó y amplió entre los siglos XVII y XVIII en Hispanoamérica "hasta convertirse en un espacio privilegiado para la experimentación, el intercambio científico y el deleite artístico" (Pérez, 2010, p.86). Según Pérez, estas transformaciones fueron el resultado por parte de las élites hispanoamericanas "de ordenar la diversidad de habitantes y territorios que debían ser convertidos en una nación” (p.86); razón por la que se emprendieron expediciones científicas con el objetivo de recoger objetos de diversos territorios para coleccionarlos $\mathrm{y}$ exhibirlos.

En Colombia, a finales del siglo XX diversos museos y programas universitarios de antropología, se vieron influenciados por los valores de plurietnicidad y multiculturalidad que promueve la Constitución Política de 1991. Por este motivo, gracias a la creación de saberes y discursos que se preocupan por conocer los orígenes de las sociedades y sus características propias, las colecciones de algunos museos están atravesadas por narrativas determinadas por el discurso oficial de estado en el marco de la construcción de nación. Esto significa encargado de realizar actos de estado por medio del ejercicio de clasificación y regulación de las poblaciones.

Según Pierre Bourdieu, el estado ejerce sus funciones por medio de agentes que realizan 'actos de estado'. Estos agentes tienen por función "permitir al grupo seguir creyendo en lo oficial, esto es, en la idea de que existe un consenso grupal sobre un cierto número de valores que son indispensables en situaciones dramáticas en las que el orden social está profundamente desafiado" (Bourdieu, 2014, p.29). Asimismo, el autor afirma que "construyen el estado, lo que significa un conjunto de recursos específicos que autoriza a sus poseedores a decir que es bueno para el mundo social como un todo, a proclamar lo oficial y a pronunciar las palabras que son, de hecho, ordenes porque están respaldadas por la fuerza de lo oficial” (p.33).

A través de las narrativas que permean las colecciones museales se produce "un juicio autorizado [que] tiene toda la fuerza del orden social, la fuerza del estado" (Bourdieu, 2014, p.11). Para Foucault, los discursos tienen consecuencias sobre la experiencia de los sujetos ya que tienen la capacidad de guiar la conducta. A pesar de que los discursos estén constituidos por enunciados, "las prácticas discursivas no son pura y simplemente modos de fabricación de discursos. Ellas toman cuerpo en el conjunto de las técnicas, de las instituciones, de los esquemas de comportamiento, de los tipos de transmisión y de difusión, en las formas pedagógicas que, a la vez, las imponen y las mantienen" (Castro, 2004, p.94). Como resultado, las narrativas de una entidad museal establecen una visión sobre el mundo, es decir, una forma de percibir e interactuar con la diferencia.

Los discursos y narrativas que se producen sobre una colección museal dotan de un sentido y características propias a los objetos que forman parte de la exhibición. De manera que, la función de los objetos se transforma puesto que, dejan de ser útiles para lo que fueron creados y se convierten en objetos coleccionables en un museo. Estos objetos son organizados en el espacio museal de tal forma que, la narrativa que los atraviesa da cuenta del mensaje que la entidad museal quiere transmitir. Por lo anterior, en el sistema de clasificación que establece el 
museo los sujetos, objetos y prácticas adquieren un valor en su relación con los demás elementos dentro del sistema.

Según Carlos Rincón, el siglo XIX conocido como el Siglo de los Museos en el mundo, se identificó "por un lado [por] el interés más creciente del público hacia el museo como institución de formación cultural, y por parte de los especialistas como lugar de investigación científica; y por otro [lado, por] el establecimiento de una nueva ciencia práctica, la museología con un objeto definido, categorías, vocabulario y metas propias" (2015, p.213). A pesar de lo anterior, en Colombia, hasta mediados del siglo XX, los museos se caracterizaron por el déficit de información sobre las piezas coleccionadas. Según Rincón, no había un diálogo entre las colecciones ni "se encontraban estudios sobre historia del arte, museografía o arqueología enfocados a dotar de información las piezas, además, no se contaba con personal profesional para, precisamente, investigar las procedencias, significados y sentidos de los objetos exhibidos en un museo" (p.213).

Por ejemplo, antes de 1938 las piezas de oro pertenecientes a sociedades prehispánicas no tenían un valor como objetos culturales, motivo por el cual no existía la necesidad de conservarlas. Ni tampoco "se las tomó por símbolos expresivos de mundos y modelos cuya valoración cultural pedía darles visibilidad pública y mucho menos se vio en ellas objetos de contemplación y goce estético" (Rincón, 2015, p.78). De hecho, no se contaba con la información documental necesaria para identificar sus características particulares, por lo que no eran consideradas índices arqueológicos, sino que su valor provenía propiamente del usufructo de la comercialización del metal. ${ }^{2}$

Aunque en la llamada Década de los museos en Colombia entre 1938 y 1948, el Museo Histórico (actualmente conocido como Museo Nacional de Colombia) y el Museo del Oro alojaban en su edificación objetos precolombinos, era escasa la información que se tenía sobre la proveniencia de estos, sus usos y características propias. No se encontraba información especializada en los rasgos culturales de las sociedades a las que los objetos prehispánicos pertenecían. Sin embargo, con la profesionalización de la antropología se empiezan a formar profesionales expertos sobre las características culturales, tradiciones y costumbres de las sociedades prehispánicas y, con la capacidad de investigar activamente dichas comunidades.

En este proceso, saberes como la antropología, la historia y la museología, entre otros, van a ser fundamentales en la constitución de la entidad museal tanto para su funcionamiento interno como para tener un marco de referencia que legitime los objetos de las colecciones; tanto así que los museos van a contar con sus propias áreas educativas. Hasta finales de la década de 1970 con la creación de departamentos educativos en el museo "la experimentación pedagógica con los patrimonios culturales musealizados empieza a traducirse lentamente en una preocupación por la construcción de políticas, marcos educativos y didácticos que potenciarán los procesos de apropiación de los acervos científicos, artísticos e históricos guardados por los museos” (López, 2013, p.21).

\footnotetext{
${ }^{2}$ El etnólogo Paul Rivet, fundador del Instituto Etnológico Nacional, entre los años de 1938 y 1939 sugirió al entonces presidente de la república Eduardo Santos, permitir al Banco de la República suministrar un porcentaje de dinero encaminado a la compra de piezas de oro precolombino "con destino a lo que se imaginaba como una sala o gabinete de exhibición" (Rincón, 2015, p.78). En consecuencia, las piezas en oro dejaron en su mayoría de ser fundidas y comercializadas en el mercado internacional y, pasaron a ser objetos de colección. Muchas de estas fueron organizadas posteriormente, en el que ahora se conoce como el Museo del Oro de Bogotá.
} 
De esta forma, nos estamos enfrentando a un doble proceso, por un lado, a la producción de valor a partir del saber y, por otro, a la transformación del significado de los objetos. En este proceso de tránsito, objetos que eran apreciados por su peso en oro adquieren un nuevo valor como objetos culturales. ${ }^{3} \mathrm{El}$ siguiente esquema de pares de oposición ${ }^{4}$ nos muestra el uso diferenciado del oro en dos periodos diferentes de la historia de Colombia. Cuando éste era fundido y comercializado en el mercado internacional y, cuando se empezaron a coleccionar las piezas precolombinas en oro.

Oro fundido / Piezas de oro precolombinas

Metal precioso / Tesoro arqueológico

De venta en el mercado internacional / Como colección en el Museo

Valor económico / Valor cultural

Mercancía / Antigüedad

De esta manera, el ejercicio pedagógico del museo consiste en conocer y caracterizar las poblaciones mediante la producción de exposiciones en las que se resaltan determinados objetos, pertenecientes a sujetos y territorios particulares o, que formaron parte de diversas prácticas. Nikolas Rose explica que "las estrategias liberales vinculan el gobierno con conocimientos positivos sobre la conducta humana desarrollados por las ciencias humanas y sociales" (Rose, 1997). Así, los saberes inscritos en las ciencias sociales se preocupan por el estudio de la vida en sus implicaciones culturales, políticas y económicas; lo cual se va a ver reflejado en las narrativas de algunos museos del país.

Por su parte, la práctica de la antropología en Colombia "produjo la necesidad de desarrollar estrategias dinámicas de proyección de tal conocimiento hacia los problemas sociales implícitos en la cuestión indígena en el país" (Echeverri, 1997, p.73). Para la formulación de soluciones viables en el proceso de la asimilación cultural de los grupos indígenas, se proponía el estudio de estas sociedades, "manteniéndose dentro de los marcos de la Academia. Esto implica un planteamiento explícito de la relación entre antropología y estado, en tanto ésta se convierte en la aplicación del saber antropológico para fines estatales, por ejemplo, a través del estudio de las instituciones indígenas para utilizarlas como órganos del gobierno local" (Echeverri, 1997, p.74). En consecuencia, la antropología se convierte en un saber de estado. Un saber, siguiendo a Foucault

es aquello de lo que se puede hablar, es una práctica discursiva que así se encuentra especificada: el dominio constituido por los diferentes objetos que adquirirán o no un estatuto científico; un saber es también el espacio en el que el sujeto puede tomar posición para hablar de los objetos de que trata en su discurso; un saber es también el campo de coordinación y de subordinación de los enunciados en los que los conceptos

\footnotetext{
${ }^{3}$ Siguiendo esta línea argumentativa, los objetos no tienen un valor intrínseco, sino que fueron sometidos a la orquestación de organizaciones y saberes que producen un "Valor cultural".

${ }^{4}$ Esquema realizado por los autores de este artículo. Si se lee de forma vertical los conceptos en cada columna se relacionan entre sí, son afines; mientras que, de forma horizontal los conceptos en cada fila de oponen entre sí. Por ello el signo “/" representa oposición. El esquema representa la concepción que se tiene de las piezas precolombinas en oro cuando eran fundidas y cuando forman parte de una colección museal.
} 
aparecen, se definen, se aplican y se transforman; en fin, un saber se define por posibilidades de utilización y de apropiación ofrecidas por el discurso. Existen saberes que son independientes de las ciencias, pero no existe saber sin una práctica discursiva definida; y toda práctica discursiva puede definirse por el saber qué forma. (Foucault, 2002, pp. 306-307)

La antropología se constituye como saber de estado ya que al ser un campo de coordinación y subordinación de los enunciados que se definen, aplican y transforman es la encargada de dar cuenta de la diversidad cultural del país, las características, prácticas y costumbres propias de una sociedad. Al ser la antropología el espacio en cual el sujeto puede tomar una posición para hablar de los sujetos que trata en su discurso, esta disciplina se posiciona con respecto a los problemas sociales y culturales de las poblaciones y territorios que estudia. Así, el estado a través de los museos como dispositivos de poder y creación simbólica incorporan en su discurso algunos contenidos de la antropología para dotar de sentido a los objetos expuestos en el museo.

La forma como los museos indagan por los orígenes de las sociedades y la domesticación de la diferencia se basa en las técnicas y formas lógicas de la antropología. Se hace, por ejemplo, investigación etnográfica y arqueología, los cuales pasan por el conocimiento de teorías como el americanismo, el evolucionismo, el particularismo histórico, la etnografía de salvamento, etc. Entonces, el museo se convierte en el lugar de consagración en el que el estado establece un discurso oficial de nación mediante la apropiación de elementos y saberes de la antropología.

El saber antropológico en este contexto corresponde a la categoría de saberes sometidos ${ }^{5}$ planteada por Foucault. Según el autor estos se caracterizan por ser

contenidos históricos que fueron sepultados, enmascarados en coherencias funcionales o sistematizaciones formales. [...]De modo que, los saberes sometidos son esos bloques de saberes históricos que estaban presentes y enmascarados dentro de los conjuntos funcionales y sistemáticos, y que la crítica pudo hacer reaparecer por medio, desde luego, de la erudición. (Foucault, 2000, p.21)

Consecuentemente, la antropología como saber sometido es utilizado y subordinado por el estado para cumplir unos propósitos específicos de conocimiento y clasificación de la diferencia. Siguiendo a Restrepo

las antropologías hegemónicas constituyen una técnica eurocéntrica para la construcción, colonización y reificación de "realidad". La especificidad de estas antropologías ha sido la domesticación de la alteridad ("cultural"- "social"); esto ha sido desplegado en un doble movimiento. Primero, y más convencionalmente, "familiarizando" la otredad; segundo, y más recientemente, exotizando la mismidad. (Restrepo, 2004, p.114)

\footnotetext{
${ }^{5}$ Con esto no queremos decir que la antropología está condenada a ser solo un saber sometido. Por el contrario, y siguiendo a Foucault, el poder es también creativo. De esta manera, la antropología y sus antropólogos encuentran maneras para generar procesos de resistencia y creación.
} 
El estado como dominante, recurre a algunos elementos de la antropología (conceptos y saberes) que le son útiles para la construcción de una narrativa sobre sí mismo y sobre la formación de nación. Los elementos que son seleccionados del saber antropológico se organizan en una sistematización formal que da sentido al discurso de estado. Marshal Berman, basado en Marx, afirma sobre el trabajo intelectual

así pues, pueden escribir libros, pintar cuadros, descubrir leyes físicas o históricas, salvar vidas, solamente si alguien con capital les paga. Pero las pretensiones de la sociedad burguesa son tales que nadie les pagara a menos que sea rentable pagarles, esto es a menos que de alguna manera su trabajo contribuya a $<<$ acrecentar el capital $>>$. Deben $<<$ venderse al detalle $>>$ aun empresario dispuesto a explotar sus cerebros para obtener una ganancia. (Berman,1991, p.115)

De forma análoga, el saber de la antropología es fragmentado, es decir, no se toma en bloque sino por partes que sirvan a la lógica del estado. Se utiliza al detalle y fraccionado para fines útiles.

La arqueología, por ejemplo, cumple un papel muy importante en este proceso de búsqueda del origen de las sociedades para la construcción de narrativas de nación. Ésta se configura como un saber sometido que, al ser el estudio sistemático de restos materiales de la vida humana ya desaparecida, amplia el conocimiento sobre las sociedades prehispánicas a través del estudio de los objetos encontrados en excavaciones arqueológicas. El museo utiliza este conocimiento para contextualizar los objetos que son exhibidos en sus salas.

La antropología provee un conocimiento sobre los orígenes de la cultura y sobre la diversidad del país, el cual el estado se encarga de dotar de un sentido propio dentro su narrativa. El museo es el encargado de establecer un discurso que organiza y le da sentido a los elementos que lo componen -narrativas, objetos, prácticas, tecnologías, sujetos, etc.-, con el fin de responder a unos intereses específicos. Este discurso tiene sus raíces en saberes especializados.

El valor que se le asigna a estos objetos es el resultado de la relación con un orden previamente establecido y otros objetos.

Los objetos que son escogidos están subordinados a los distintos mensajes que el museo quiera plasmar y difundir. Es por este motivo que entre los cientos o miles de piezas con los que cuentan algunos museos, los curadores junto con el equipo encargado de montar una exposición tienen que saber escoger las piezas adecuadas para mostrar claramente el mensaje que el museo quiere transmitir mediante una exposición. (Sánchez y Ospina, 2017)

La creación de saberes encargados del estudio y comprensión de diferentes sociedades, se produjo en un contexto en el cual "el conocimiento experto se convierte en un recurso fundamental para configurar e interpretar el mundo" (Escobar, 1993, p.386). Según Escobar "expertos de todo tipo proveen las monedas [saberes, formas de entender el mundo, normatividades, etc.] con las cuales el estado y las clases dominantes articulan las 'necesidades' de la gente, convirtiéndolas en objetos de administración del estado" (p.386). De acuerdo con lo anterior, los museos establecen sistemas de clasificación que organizan la percepción sobre unos 
sujetos, objetos, lugares, eventos y acontecimientos a través de las narrativas que dotan de sentido a las colecciones.

¿Cómo se instaura una percepción particular sobre la alteridad? Se ejerce una regulación sobre la concepción de la otredad en la medida que se inserta en un sistema de clasificaciones y relaciones propio del saber dominante. Los expertos encargados estudian lo que caracteriza a una población, los objetos y las prácticas que forman parte de su cotidianidad. Es necesario reiterar para evitar confusiones o malinterpretaciones, que no se trata de que la antropología haya surgido en función del estado para promover la construcción de nación mediante unos parámetros específicos, como si los intereses del Estado fueran los intereses del saber antropológico, más bien sucede que el estado de forma estratégica adopta algunos elementos de la antropología que le son útiles en su discurso.

En síntesis, así como la antropología ha proveído conocimientos sobre la diversidad cultural del país, el museo ha sido el encargado de instaurar una narrativa que orienta, organiza y da sentido a los elementos a los que se refiere -sujetos, objetos, lugares, saberes, tecnologías, discursos y prácticas. En el proceso de construcción de nación por parte del estado, el saber antropológico ha sido comprendido como un saber-poder que por medio de los museos y, de la mano de los conocimientos del saber antropológico ha fijado una visión particular sobre la diversidad cultural del país.

El campo museal es tan sólo uno de los campos en los cuales el saber antropológico ha tenido una influencia directa, no sólo en los aspectos que en este artículo mencionamos, sino en otros más. Para tener una visión panorámica sobre los usos sociales de esta disciplina sería importante realizar un análisis de los aportes de la antropología en los campos de la salud, la educación, el arte, la ecología, el sector financiero, jurídico, político, etc. Sería oportuno conocer cómo las dinámicas del mercado afectan a la antropología en cada uno de estos campos, lo anterior teniendo en cuenta los cambios que ha atravesado esta disciplina, incluyendo su proliferación en el sector público y, recientemente, en el privado.

\section{Bibliografía}

Berman, Marshal (1991) Todo lo solido se desvanece en el aire. La experiencia de la modernidad. Buenos aires. Siglo XXI

Buenaventura (2008). "Expertos culturales e intervención social. Tensiones y transformación en antropología aplicada". Revista $C S$, Universidad ICESI.

Castro, Edgardo (2004). El vocabulario de Michel Foucault. Un recorrido alfabético por los temas, conceptos y autores. Buenos Aires, Argentina. Universidad Nacional de Quilmes.

Durkheim, Emile (1982) Las reglas del método sociológico. Editores Orbis, Barcelona.

Echeverry, Marcela (1997). "El proceso de profesionalización de la Antropología en Colombia. Un estudio de caso en torno a la difusión de las ciencias y su institucionalización”. Historia Crítica, 15, pp. 67-79. 
Escobar, Arturo (1993). The Limits of Reflexivity: Politics in Anthropology's Post-Writing Culture Era. En Journal of Anthropological Research, 49, pp 377-391.

Foucault, Michel (2002). La arqueología del saber. Buenos Aires, Argentina. Siglo XXI. Jimeno y otros (1993) "Evaluación de la producción y practica antropológica. Parámetros y tendencias en la formación profesional”. En Informes Antropológicos, ICANH. Bogotá, Pp 33-66.

López, William (2013). Museo en tiempos de conflicto: memoria y ciudadanía en Colombia. Bogotá, Colombia. Universidad Nacional de Colombia.

Ospina, Juan Camilo (2018). Tesis de pregrado. Confesiones impersonales, un análisis estructural del campo antropológico en la Pontificia Universidad Javeriana. Pontificia Universidad Javeriana, Bogotá, Colombia.

Pérez, Amada (2010). "Hacer visible, hacerse visibles: la nación representada en las colecciones del museo. Colombia, 1880-1912”. En Memoria y Sociedad. 14 (28): 85-106

Pineda Camacho, Roberto (2008). "El Departamento de Antropología de la Universidad de Los Andes cumple sus primeros Cuarenta años". Universitas Humanística 66: 19- 24.

Pineda Giraldo, Roberto (1999). "Inicios de la Antropología en Colombia". Revista De Estudios Sociales.

. (2005). "La enseñanza y los campos de la antropología en Colombia". Universitas Humanísticas 59, Bogotá.

Restrepo, Eduardo (2004). "Antropologías en el mundo". Revista de Antropología Jangwa Pana, 3, Universidad del Magdalena

Rincón, Carlos (2015). Avatares de la memoria cultural en Colombia Formas simbólicas del Estado, museos y canon literario. Bogotá, Colombia. Editorial Pontificia Universidad Javeriana.

Rose, Nikolas (1997) "El gobierno en las democracias liberales "avanzadas" del liberalismo al neoliberalismo". Archipiélago: Cuadernos de crítica de la cultura, ISSN 0214-2686, N. 29, págs. 25-40.

Sánchez y Ospina (2017). El reto de incluir las narrativas de las minorías a los museos. Las2Orillas [Online]. Obtenido de: https://www.las2orillas.co/el-reto-de-incluir-lasnarrativas-de-las-minorias-los-museos/

Sánchez, Valeria (2019). El museo como dispositivo de poder. Construcciones simbólicas en torno al proceso de paz en Colombia. Tesis de pregrado. Pontificia Universidad Javeriana. Bogotá, Colombia.

Zambrano Escovar, M., \& Durán Urrea, M. M. (2017). "El efecto espejo: sexo, género y cuidado en las trayectorias profesionales y las jerarquías morales y laborales de la antropología en Colombia. Revista Colombiana De Sociología, 40(2), 87.

doi:10.15446/rcs.v40n2.66386 